\title{
Complications Following Dental Extractions in a Mobile Dental Clinic
}

\author{
Manor $\mathrm{Y}^{*}$, Mardinger $\mathrm{O}^{2}$, Zaks $\mathrm{O}^{3}$, Haim $\mathrm{D}^{4}$, Manor $\mathrm{A}^{5}$ and Chaushu $\mathrm{G}^{6}$
}

${ }^{1}$ Department of Oral and Maxillofacial Surgery, The Maurice and Gabriela Goldschleger School of Dental Medicine, Aviv University \& clinic of Oral Medicine and dental implantation Surgery, Assaf Harofe Medical Center Zriffin, Israel

${ }^{2}$ Department of Oral and Maxillofacial Surgery, The Maurice and Gabriela Goldschleger School of Dental Medicine, Tel-Aviv University, Israel \& Department of Oral and Maxillofacial Surgery, Sapir Medical Center Kfar Saba, Israel ${ }^{3}$ Departments of Oral and Maxillofacial Surgery, The Maurice and Gabriela Goldschleger School of Dental Medicine, Tel-Aviv University, Israel

${ }^{4}$ Clinic of Oral Medicine and dental implantation Surgery, Assaf Harofe Medical Center Zriffin, and the Mobile Dental clinic of Oral Health, Israel

${ }^{5}$ Administration Edith Wolfson Medical Center Holon Israel

${ }^{6}$ Departments of Oral and Maxillofacial Surgery, Beilinson Medical Center Petah Tikva, Departments of Oral and Maxillofacial Surgery, The Maurice and Gabriela Goldschleger School of Dental Medicine, Tel-Aviv University, Israel

${ }^{*}$ Corresponding author: Manor Y, Department of Oral and Maxillofacial Surgery, School of Dental Medicine, Aviv University, Israel, Tel: 972-50-8560114, E-mail: yifatmanor@gmail.com

Citation: Manor Y, Mardinger O, Zaks O, Haim D, Manor A, et al. (2015) Complications Following Dental Extractions in a Mobile Dental Clinic. J Dent Oral Care Med 1(1): 101. doi: 10.15744/2454-3276.1.101

Received Date: January 07, 2015 Accepted Date: May 12, 2015 Published Date: May 14, 2015

\begin{abstract}
Objectives: To assess the incidence of possible intraoperative and post-operative complications following dental extractions in mobile dental treatment systems.

Study design: Medical files of 51 individuals treated in a mobile dental clinic (study group) and 60 individuals treated in a conventional dental clinic (control group) between the years 2008-2011 were evaluated retrospectively. Demographic and clinical data, intraoperative and postoperative complications and their management were considered. Patients over 60 years who underwent dental extractions were evaluated for immediate complications and postoperative complications.

Results: 111 patients met the inclusion criteria, 54 males and 57 females. The mean age was 77 in the study group and 69 in the control group. The patients underwent 162 dental extractions. Most of the patients had neurologic and cognitive diseases like dementia, Parkinson, $\mathrm{s} / \mathrm{p}$ brain stroke as well as hypertension and diabetes. Indications for extractions included hopeless teeth, periodontitis and dental caries, there was no need for bone removal or flap elevation. The rate of minor complications was found in $10 \%$ of the patients intraoperatively and postoperatively. All the complications were solved on the spot by the dental staff. None of the patients had life threatening complications or other conditions requiring hospital based treatments.
\end{abstract}

Conclusion: In carefully selected cases such as controlled high blood pressure, controlled neurologic disorders and controlled diabetes, dental extractions can be performed in a mobile dental clinic with minimal minor complications.

Keywords: Oral surgery mobile dental unit; Complications

\section{Introduction}

The developments in the medicinal field led to demographic changes characterized by a growth in the elderly population. The proportion of patients with mobility limitations is higher in this population. Since the need for dental treatments does not skip any population group, a special facility had to be developed in order to solve problems of availability and accessibility [1].

The mobile clinic was designed to give dental treatments in all fields for medically stable but handicapped patients. These patients suffer from dental negligence and the need for extractions logically rises with the age. The mobile dental clinic should provide several dental treatments including extractions. The patients get their treatments at bed side at their home instead of transporting them. The aim of the planned treatments is to give these patients the optimal solution for their needs with minimal risks and complications and minimal chance for re-treatments [1-4].

The mobile dental clinic was designed to enable dental treatments for the homebound, individuals over the age of 18 who are unable to reach a simple dental clinic. Routine treatments include plaque removal, scaling, fillings, root canal therapy, regular and surgical extractions, repairing full and partial dentures etc. [5]. 
There is great variability in physical, medical, and mental health status among people over the age of sixty-five years. They are defined by the Bureau of Health Professions as "elderly" and consist of "a population with health care conditions and needs which differ significantly from those of younger people, which are often complicated by the physical, behavioral, and social changes associated with aging. Declines in functional status were found to be negatively associated with dental services use $[5,6]$. Handicapped patients unable to arrive to conventional clinics and observe a fair oral hygiene, suffer more and more teeth have to be extracted.

Tooth extractions may be followed by several known complications [7-10]. Among intraoperative complications encountered the following are included: needle breaks, bleeding, crown or root fractures, damage to other teeth, alveolar fractures, jaw fractures, soft tissue abrasions, gingival tears, foreign body aspirations, tooth displacement into the maxillary sinus or soft tissue and other spaces and pain.

Immediate post-operative complications include: bleeding, pain, infections, hematomas, soft tissue emphysema. Late postoperative complications include: epulis granulomatosum, trismus, pain, paraesthesias, temporomandibular joint disorders, sinus communications, and alveolar osteitis. There is a higher risk for such complications due to the inconvenient circumstances and due to the medical complexity of patients treated at bedside.

The purpose of our study was to find out if there are more complications following surgical extractions performed by the mobile dental clinic compared to conventional dental clinics and to identify the possible risk factors for these complications.

\section{Patients and Methods}

A retrospective cohort study was performed and data was collected from patients' files and organized in a structured questionnaire. The study group included patients who were treated by a mobile dental clinic staff, the control group included patients who were treated in a conventional clinic between the years 2008-2011. The study was approved by the ethical committee of the University.

The inclusion criteria were:

1. Patients over sixty years

2. Patients who underwent surgical extraction by the mobile dental clinic

3. Complete data regarding the systemic conditions details of the surgical treatment and the follow up of at least one month postoperatively.

Exclusion criteria of patients:

1. High blood pressure (over 160/100).

2. Recent MI

3. Signs of $\mathrm{CHF}$

4. Uncontrolled movements (Parkinson)

5. Non cooperative patients.

The information collected included demographic and clinical data (age, gender, systemic condition). Preoperative assessment and clinical diagnosis of the extracted tooth and pathological findings. All medical treatments of the patients, mainly anticoagulants, were not discontinued before the surgery.

All intraoperative and postoperative complications were recorded from the files. Complications like exaggerated bleeding, pain during the surgery, root fracture and or displacement. Postoperative complications like bleeding, infection, acute pain, alveolitis, swelling and late post operative complications like residual broken root, epulis granulomatosum, trismus, swelling and edema.

Statistical workup included analysis of variance with repeated observations. Differences between the groups by T-Test, chi square test, Fisher exact test and Mann-Whitney test. $\mathrm{X}<0.005$ was determined as statistically significant.

\section{Results}

51 patients underwent 161 tooth extractions at the mobile dental unit setup at bedside in the nursing institution. Karnofsky score was: 30 in 16 patients, 40 in 17 patients and 50 in 18 patients. 60 patients underwent 70 extractions at a conventional clinic all of them were 100 at karnofsky score.

The mean age was 77 years old and 69 years old in the study and the control group respectivel. There was a slight higher percentage of women, 55\%. Most of the patients in both groups underwent up to two extractions per session. The extracted teeth were simple rooted or multi rooted but mostly residual roots. The medical diagnoses of the patients are presented in table 1.8 patients suffered from more than one medical problem and hypertension was the most frequent. Medical treatment- presented in table 2.21 patients on anticoagulants-Aspirin-19 patients, coumadin- 1 patient, plavix- 1 patient.

Complications- there were only 6 cases of minor complications intraoperatively and post operatively. The intraoperative complications are presented in table 2. No events of bleeding, or uncontrolled situation in the local and systemic condition were found. All the complications were solved on the spot (dental clinic/bedside) by the dental staff and did not require further intervention. 


\begin{tabular}{|c|c|c|c|c|c|c|}
\hline Parkinson Disease & Dementia & Stroke & Osteoporosis & Hypothyroidism & Hypertension & $\begin{array}{c}\text { Diabetes } \\
\text { Type 1 \& 2 }\end{array}$ \\
\hline 7 & 8 & 9 & 3 & 2 & 22 & 7 \\
\hline
\end{tabular}

Table 1: Medical diagnoses in the study group

\begin{tabular}{|c|c|c|c|}
\hline Group & Aspirin & Coumadin & plavix \\
\hline Study & 19 & 1 & 1 \\
\hline Control & 4 & 2 & 0 \\
\hline
\end{tabular}

Table 2: Anticoagulant medical treatment

\begin{tabular}{|c|c|c|c|}
\hline Group & Soft tissue Damage & Pain & Roots fracture \\
\hline Study & 2 & 1 & 2 \\
\hline Control & 2 & 2 & 2 \\
\hline
\end{tabular}

Table 3: Intraoperative complications

\section{Discussion}

With the increase in life expectancy the number of older patients requiring dental treatments increases. Most of those patients who had mobility problems do not have the possibility to receive dental treatments in conventional clinic and receive the assistance of at least one person for their mobilization. Moreover since handicapped patients do have problems with oral hygiene such as tooth brushing the use of dental cleaning devices leading to further loss of teeth, most of their treatments are extraction of lost teeth for removable denture. Those patients will benefit from treatments at bedside without mobilizing them to a clinic which has the economical and psychological benefits [11].

Among those patients who are medically stable and can be cooperative dental treatments and dental extractions can also be performed bedside by mobile dental unit.

In this study we showed that the overall rate of complications following extractions of older immobile people is low (10\%) even though the less convenient circumstances at mobile dental clinic. According to Baniwal study [12]: The rate of complications following dental extractions is $1 \%$. Of the complications $58 \%$ were intraoperative, $41 \%$ postoperative, $1 \%$ late postoperative. The difference in the rate of complications in our study is explained by the fact that our patients mean age was higher (over 60 years) and by a small sample of study.

It was shown that homebound elderly people reported greater difficulties than non-homebound people in communication, eating, relaxation, and life satisfaction as related to oral health [13]. Homebound dental patients who receive dental treatment bedside have higher quality of life and that Elderly people with needs of supportive care have lost many teeth before they become dependent. Health promotion should be a priority in early ageing populations to prevent oral diseases and tooth loss [14].

Bleeding is one of the known complications during or following extraction. No event of uncontrolled or exaggerated bleeding was found although continuation of anticoagulant therapy. Careful case selection and good skill of the dental staff could enhance it. Brennan et al found that there is no need of discontinuation of Aspirin therapy for tooth extraction no excess oral bleeding was found in those patients [15].

Corporate dental clinics prefer to refer patients with parkinson, demention and other neurological problems to hospital treatment by general anesthesia. The reason is probably the chair time which is expensive and the alternative of using it for more rentable procedures and patients. However on the other side the economical consideration of mobilizing the patients for dental treatments in hospitals cannot be ignored.

Dental practitioners in corporate clinics prefer to refer medically stable compromised patients to hospital treatment. Those patients can be treated even bedside without any complications. Dental practitioners should consider simple dental extractions in their clinic instead of their referral as long as they have the good skill, the patient disability is controlled and the surgical procedure is not complicated.

It was found by Gluzman et al that the oral health status of these homebound elderly was poor and their quality of life was significantly affected by the lack of basic dental care [16]. According to that and to our findings we think that patients with mobility problem and stable medical condition with uncomplicated extractions could be treated bedside. All the complications developed intraoperatively and postoperatively were minimal without endangering the clinical situation or additional requirement for hospital treatment. The reason may be a careful case selection for treatment by mobile dental treatment and good skill of the treatment staff.

\section{Conclusion}

Dental extraction in stable medically compromised homebound patients with a good control of their medical situation can be performed at bedside without a high risk of major complications. There are medical advantages to bed side dental treatments and the authors propose that the economic advantages should be investigated. 


\section{References}

1. Chalmers JM, Ettinger RL (2008) Public health issues in geriatric dentistry in the United States. Dent Clin North Am 52: 423-46.

2. Hurst PS, Noblett WC (1990) Geriatric dentistry. Otolarygol Clin North Am 23: 1097-107.

3. Syrjälä AM, Ylöstalo P, Ruoppi P, Komulainen K, Hartikainen S, et al. (2012) Dementia and oral health among subjects aged 75 years or older. Gerodontology 29: 36-42.

4. Peltola P, Vehkalahti MM, Wuolijoki-Saaristo K (2004) Oral health and treatment needs of the long-term hospitalised elderly. Gerodontology 21: 93-9.

5. Lee EE, Thomas CA, Vu T (2001) Mobile and portable dentistry: alternative treatment services for the elderly. Spec Care Dentist 21: 153-5.

6. Shay K (1994) Identifying the needs of the elderly dental patient. The geriatric dental assessment. Dent Clin North Am 38: 499-523.

7. Boulux GF, Steed MB, Percianccante VJ (2007) Complications of third molar surgery. Oral Maxillofac Surg Clin North Am 19: 117-28.

8. Brauer HU (2009) Unusual complications associated with third molar surgery: A systematic review. Quintessence Int 40: 565-72.

9. Osaki T, Nomura Y, Hirota J, Yoneda K (1995) Infections in elderly patients associated with impacted third molars. Oral Surg Oral Med Oral Pathol Oral Radiol Endod 79: 137-41.

10. Migliorati , Siegel MA, Elting LS (2006) Bisphosphonate-associated osteonecrosis: a long-term complication of bisphosphonate treatment. Lancet Oncol 7: 508-14.

11. Dougall A, Fiske J (2008) Access to special care dentistry, part 9. Special care dentistry services for older people. Br Dent J 205: 421-34.

12. Baniwal S, Pauder KR, Pyakurel U, Bajracharia M, Niraula SR (2007) Prevalence of complications of simple tooth extractions and its comparison between a tertiary center and peripheral centers: a study conducted over 8,455 tooth extractions. JNMA J Nepal Med Assoc 46: 20-4.

13. Zini A, Sgan-Cohen HD (2008) The effect of oral health on quality of life in an underprivileged homebound and non-homebound elderly population in Jerusalem. J Am Ger Asssoc 56: 99-104.

14. Holmén A, Strömberg E, Hagman-Gustafsson ML, Wårdh I, Gabre P (2012) Oral status in home-dwelling elderly dependent on moderate or substantial supportive care for daily living: prevalence of edentulous subjects, caries and periodontal disease. Gerodontology 29: 503-11.

15. Brennan MT, Valerin MA, Nol JL, Napenas JJ, Kent ML, et al. (2008) Aspirin Use and Post-operative Bleeding from Dental Extractions. J Dent Res 87: 740-4.

16. Gluzman R, Meeker H, Agrawal P, Patel S, Gluck G, et al. (2013) Oral health status and needs of homebound elderly in an urban home-based primary care service. Spec Care Dentist 33: 218-26.

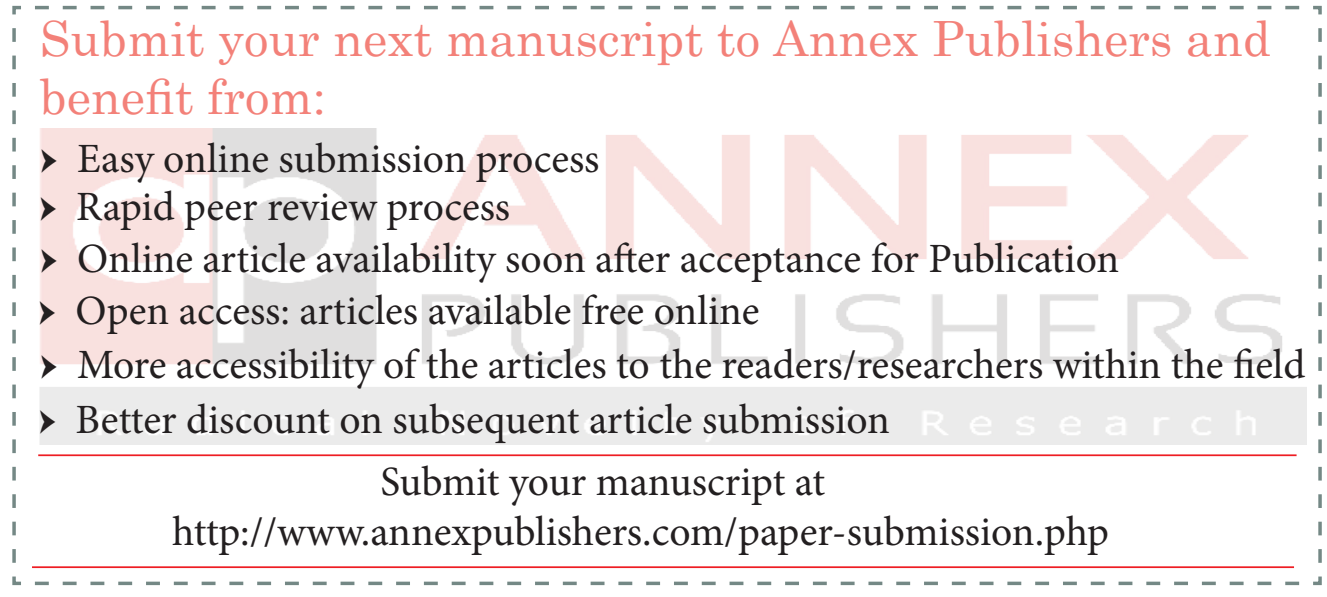

